\section{THE NEW ALGEBRAS}

$\mathrm{P}$ ROF. E. T. WHITTAKER'S presidential address to the Royal Society of Edinburgh, delivered on Oetober 25, on "The New Algebras and their Significance for Physics and Philosophy", contains a characteristically lucid account of the main features of non-commutative algebra and its application to modern physics. Its significanre for philosophy follows perhaps by implication; but it is to be regretted that, presumably through limitation of space, this interesting aspect of the subject received no specific treatment.

It is appropriate that the occasion of the address should fall almost exactly a hundred years after the discovery of quaternions by Hamilton, for this event, as Prof. Whittaker points out, marked the beginning of the emancipation of algebra from the confines of classical ideas, according to which algebraic symbols necessarily okeyed the rules of numbers. Quaternions were devised to represent operations (actually operations of spatial rotation, but the general principle involved is capable of much wider application), and the order of performance of two operations, unlike the order of multiplication by two numbers, has in general an effect on the result obtained. In the developments of the new idea, such as Boolean algebra, Pierce's logic of relatives, Whitehead and Russell's logic, and Cayley's matrix algebra, the symbolism traditionally employed for elementary processes with numbers is adopted for different, though somewhat analogous, processes with operators, so that, for example, $a b C$, which in classical algebra stands for the product of $a, b$ and $C$ and is identical with $b a C$, stands in operational algebra for the operation $b$ performed on $C$ and the operation $a$ on the result. It is not, in this case, normally equivalent to $b a C$.

Prof. Whittaker gives some interesting examples of this generalization of symbolic processes, and then introduces the conceptions of modern physics in which scope has been found for its application. The older physics sought to interpret physical events in terms of moving mass-points, and for this interpretation the algebra of numbers was adequate. The refined experiments now possible, however, show that such an interpretation is not admissible for the extremely small entities with which modern physics is concerned. We can no longer think of those entities as particles. "We have no right to postulate the existence of entities which lie beyond the knowledge actually obtainable by observation, and which have no part in the prediction of future effects. Thus the classical concept of a particle must be discarded ; in its stead there has been introduced a new fundamental element in the description of the external world, which is called a state". States are "events which extend over more than one point of space and more than one instant of time and which yet are ultimate events; that is, they cannot be analysed. into anything simpler than themselves".

For the representation of states, numbers are inappropriate. The position and momentum of an ultimate physical entity can no longer be regarded as separate quantities, each specifiable by its intrinsic numerical measure. They have to be taken together and represented by a joint symbol, and the problem is to find symbols the rules of manipulation of which fit them to represent the physical situations which actually occur. It turns out that a new algebra of the type introduced by Hamilton, in which 'multi- plication is not commutative, meets the case ; it is the matrix algebra of Cayley. This algebra "is, in fact, the symbolism appropriate to things that cannot be measured exactly", although, of course, all observable results obtained by its use must agree with the facts of observation.

Prof. Whittaker gives an example to show how the idea of an operator may be regarded as an extension of the idea of an ordinary number, thus justifying the use of the word 'multiplication' for what are essentially two different processes. In this connexion an illustration occurring in Kelland and Tait's: "Introduction to Quaternions" is worth recalling. (Incidentally, Prof. Whittaker speaks appreciatively of Tait's efforts to make known the importance of quaternions.) The extension of the idea of multiplication from whole numbers to fractions involved a generalization as difficult to understand in its day as the extension to operations now is. Originally, multiplication implied a process of addition; to multiply by 3 was to add three numbers together. 'Multiplication' by $\frac{1}{2}$, however, is in no sense an addition, and the result is, in fact, to give a smaller number. This is, in essence, as paradoxical as the non-commutability of matrix multiplication.

\section{N.Z. DEPARTMENT OF SCIENTIFIC RESEARCH}

$T$ HE seventeenth annual report of the Department of Scientific and Industrial Research, New Zealand, covering the year 1941-42, includes the Minister's statement, the secretary's report, and reports of the various research committees of the Council, on research work at the agricultural colleges, on the work of the Dominion Laboratory, the Dominion Observatory, the Dominion Physical Laboratory, and the Geological Survey, as well as of the Magnetic Observatory, Christchurch.

The Department's activities have continued to be concentrated on problems arising out of the War, but both the Dominion Physical Laboratory and the Radio Development Laboratory have been established in improved buildings and provided with additional skilled staff to give greater assistance to the three Fighting Services and the munitions industry. Important defence scientific units have been established at the University of Otago, and at Auckland and at Canterbury University Colleges. The first commercial dehydration plants for butter, vegetables and meat came into operation during the year, and the plants are giving very satisfactory dried foodstuffs, largely because of the scientific effort put into their design and to previous testing on pilot plants by the staff of the Department. Investigations have been extended to examination of the raw materials used, their pre-treatment to conserve their food values and vitamin contents, and to their packaging in a form suitable to retain high quality when used as army rations by troops operating in difficult field conditions. Work on an up-to-date fruit dehydrating plant is well advanced.

Maps showing the soil types occurring in the North Island have been completed, and, based on these maps, others showing soils which will respond to lime treatment in different degrees have been prepared. A map of the areas suffering from erosion in the mountainous region of the South Island has been completed, and soil surveys have been made of three 